\title{
SCATTERED PRODUCTS IN FUNDAMENTAL GROUPOIDS
}

\author{
JEREMY BRAZAS
}

\begin{abstract}
Infinitary operations, such as products indexed by countably infinite linear orders, arise naturally in the context of fundamental groups and groupoids. We prove that the well-definedness of products indexed by a scattered linear order in the fundamental groupoid of a first countable space is equivalent to the homotopically Hausdorff property. To prove this characterization, we employ the machinery of closure operators, on the $\pi_{1}$-subgroup lattice, defined in terms of test maps from one-dimensional domains.
\end{abstract}

\section{Introduction AND Results}

1.1. Introduction. The are many natural situations where a group $G$ with some additional structure admits an infinitary operation such as an infinite product, i.e. elements $\prod_{n=1}^{\infty} g_{n} \in G$ assigned to certain $\omega$-sequences $\left\{g_{n}\right\} \in G^{\omega}$, where product values satisfy $\prod_{n=1}^{\infty} g_{n}=\left(g_{1} g_{2} \cdots g_{k}\right) \prod_{n=k+1}^{\infty} g_{n}$ for all $k \in \mathbb{N}$. For example, the existence and uniqueness of classical infinite sums and products in $\mathbb{R}$ depends on the topology of $\mathbb{R}$. Non-abelian groups and groupoids with infinitary operations arise naturally in the context of "wild" algebraic topology, in particular, the study of fundamental group(oid)s of spaces with non-trivial local homotopy. The welldefinedness of such products in fundamental group(oid)s of one-dimensional and planar sets plays an important role in Katsuya Eda's homotopy classification of one-dimensional Peano continua [15] and related "automatic continuity" results for fundamental groups of one-dimensional and planar Peano continua [8, 12, 22.

The fundamental group $\pi_{1}$ with its familiar binary operation is the skeleton of the fundamental groupoid $\Pi_{1}$ and, hence, determines it's composition operation. However, the determination of infinitary operations does not not pass so easily from groups to groupoids since infinitary products in $\pi_{1}(X, x), x \in X$ depend on pointlocal structure and only partially inform infinitary products in $\Pi_{1}(X)$, which may depend more on the global structure of $X$.

In the context of fundamental groups (resp. groupoids), infinite product values are defined in terms of the topology of representing loops (resp. paths): if $\alpha_{n}$ is a sequence of loops (resp. paths), then the $\omega$-product $\prod_{n=1}^{\infty}\left[\alpha_{n}\right]$ is the homotopy class of the infinite concatenation $\left(\alpha_{1} \cdot\left(\alpha_{2} \cdot\left(\alpha_{3} \cdot(\cdots)\right)\right)\right.$ ) when the concatenation is defined and continuous. The $\omega$-product operation on homotopy classes is welldefined if path-homotopic factors result in path-homotopic infinite concatenations. There are subspaces of $\mathbb{R}^{3}$ for which this is not the case (see Example 5.13).

Since the components of an open set of $[0,1]$ may have any countable linear order type, one may also define group(oid) products $\prod_{i \in \mathscr{L}}\left[\alpha_{i}\right]$ indexed by any countable linear order $\mathscr{L}$. The primary purpose of this paper is to characterize those spaces

Date: September 17, 2019. 
whose fundamental groups and/or groupoids admit well-defined $\omega$-products and, more generally, well-defined products over scattered linear orders.

1.2. Main Results. To effectively formalize the well-definedness of products indexed over linear orders, we employ the fundamental group of the usual Hawaiian earring space $\mathbb{H}$ and the loop $\ell_{n}:([0,1],\{0,1\}) \rightarrow\left(\mathbb{H}, b_{0}\right)$ traversing the $\mathrm{n}$-th circle. The group $\pi_{1}\left(\mathbb{H}, b_{0}\right)$ is isomorphic to a locally free group of reduced words where the letters of each word are indexed by a countable linear order and each letter $\left[\ell_{n}\right]$ and it's inverse may appear at most finitely many times in each word [6, 11. The subgroup of scattered words $S c \leq \pi_{1}\left(\mathbb{H}, b_{0}\right)$ consists of homotopy classes of loops $\alpha:([0,1],\{0,1\}) \rightarrow\left(\mathbb{H}, b_{0}\right)$ for which $\alpha^{-1}\left(b_{0}\right)$ is a scattered set, or equivalently, for which the components of $[0,1] \backslash \alpha^{-1}\left(b_{0}\right)$ have a scattered order type (see [13]). In what follows, $\pi_{1}$ and $\Pi_{1}$ refer to the fundamental group and groupoid respectively.

Definition 1.1. Let $X$ be a path-connected space. We say that $X$ has

(1) well-defined infinite $\pi_{1}$-products if for any $x \in X$ and maps $f, g:\left(\mathbb{H}, b_{0}\right) \rightarrow$ $(X, x)$ such that the induced homomorphisms $f_{\#}, g_{\#}: \pi_{1}\left(\mathbb{H}, b_{0}\right) \rightarrow \pi_{1}(X, x)$ agree each individual letters, i.e. $f_{\#}\left(\left[\ell_{n}\right]\right)=g_{\#}\left(\left[\ell_{n}\right]\right)$ for all $n \in \mathbb{N}$, then $f_{\#}$ and $g_{\#}$ also agree on the standard infinite product $\left[\ell_{1} \cdot\left(\ell_{2} \cdot\left(\ell_{3} \cdot(\cdots)\right)\right)\right]$.

(2) well-defined scattered $\pi_{1}$-products if for any $x \in X$ and maps $f, g:\left(\mathbb{H}, b_{0}\right) \rightarrow$ $(X, x)$ such that $f_{\#}\left(\left[\ell_{n}\right]\right)=g_{\#}\left(\left[\ell_{n}\right]\right)$ for all $n \in \mathbb{N}$, then $f_{\#}$ and $g_{\#}$ also agree on the subgroup $S c \leq \pi_{1}\left(\mathbb{H}, b_{0}\right)$ of scattered words.

(3) well-defined infinite $\Pi_{1}$-products if for any paths $\alpha, \beta:[0,1] \rightarrow X$ that agree on $S=\left\{\frac{n-1}{n} \mid n \in \mathbb{N}\right\} \cup\{1\}$ and such that $\left.\alpha\right|_{[a, b]}$ is path homotopic to $\left.\beta\right|_{[a, b]}$ for each component $(a, b)$ of $[0,1] \backslash S$, then $[\alpha]=[\beta]$ in $\Pi_{1}(X)$.

(4) well-defined scattered $\Pi_{1}$-products if for any paths $\alpha, \beta:[0,1] \rightarrow X$ that agree on a closed scattered set $S \subset[0,1]$ containing $\{0,1\}$ and such that $\left.\alpha\right|_{[a, b]}$ is path homotopic to $\left.\beta\right|_{[a, b]}$ for each component $(a, b)$ of $[0,1] \backslash S$, then $[\alpha]=[\beta]$ in $\Pi_{1}(X)$.

The homotopically Hausdorff property (see Definition 5.1) is the most fundamental point-local obstruction to applying shape theoretic methods [10, 16, 18] and generalized covering space methods [1, 4, 19, 20, to study fundamental groups of spaces that lack a simply connected covering space. Spaces known to be homotopically Hausdorff include those spaces whose fundamental group naturally injects into the first shape homotopy group [19, including all one-dimensional spaces, planar sets, and certain inverse limits of manifolds. Additionally, many other examples exhibiting more intricate infinite $\pi_{1}$-product operations are homotopically Hausdorff, e.g. $A, B \subset \mathbb{R}^{3}$ in [9], the space $R X$ in [24], and the "Hawaiian pants" space $\mathbb{P} \subset \mathbb{R}^{3}$ in [3. We refer to [2, 9, 17] for characterizations and comparisons of the homotopically Hausdorff property with other local properties. The main result of this paper is the following theorem.

Theorem 1.2. For any path-connected first countable space $X$, the following are equivalent:

(1) $X$ has well-defined infinite $\pi_{1}$-products,

(2) $X$ has well-defined scattered $\pi_{1}$-products,

(3) $X$ has well-defined infinite $\Pi_{1}$-products,

(4) $X$ has well-defined scattered $\Pi_{1}$-products,

(5) $X$ is homotopically Hausdorff. 
Obviously, $(4) \Rightarrow(3) \Rightarrow(1)$ and $(4) \Rightarrow(2) \Rightarrow(1)$ since loops are paths and $\omega$ is a scattered order. The primary difficulty in the proof of Theorem 1.2 is to verify the equivalence of (2) and (4) with the other properties. In fact, we prove a subgrouprelative version of $(4) \Leftrightarrow(5)$, which applies to arbitrary subgroups of $\pi_{1}\left(X, x_{0}\right)$ (see Theorem 6.6). To achieve this, we utilize the closure operator framework introduced in 2. In particular, we compute subgroup closures (see Theorem 5.10 and Corollary 5.11) by applying the theory of reduced paths in one-dimensional spaces [5] and adapting the proof of Hausdorff's characterization of scattered linear orders (see Chapter 5 of [23]).

The remainder of this paper is structured as follows: In Section 2, we set up notation and recall relevant background on paths and linear orders. In Section 3, we recall the closure operators on the $\pi_{1}$-subgroup lattice from [2] and introduce an inductive construction of these closures. In Section 4, we introduce the scattered extension $\operatorname{Sc}(H)$ of a subgroup $H \leq \pi_{1}\left(X, x_{0}\right)$ and relate it to closure under infinite products (see Theorem 4.7). In Section 5, we recall the relative homotopically Hausdorff property and use the scattered extension to prove the equivalence (1) $\Leftrightarrow(2) \Leftrightarrow(5)$ in Theorem 1.2 (see Theorem 5.12). In Section 6, we introduce a closure operator that allows us to establish the equivalence of (3) and (4) with the other three properties in Theorem 1.2 (see Remark 6.8). We conclude the paper, in Section 7, with a brief remark on products indexed by arbitrary countable order types.

\section{PRELIMINARIES AND NOTATION}

Throughout this paper, $X$ will denote a path-connected topological space and $x_{0} \in X$ will be a basepoint. The homomorphism induced on $\pi_{1}$ by a based map $f:(X, x) \rightarrow(Y, y)$ is denoted $f_{\#}: \pi_{1}(X, x) \rightarrow \pi_{1}(Y, y)$.

A path is a continuous function $\alpha:[0,1] \rightarrow X$, which we call a loop based at $x \in X$ if $\alpha(0)=\alpha(1)=x$. If $[a, b],[c, d] \subseteq[0,1]$ and $\alpha:[a, b] \rightarrow X, \beta:[c, d] \rightarrow X$ are maps, we write $\alpha \equiv \beta$ if $\alpha=\beta \circ \phi$ for some increasing homeomorphism $\phi$ : $[a, b] \rightarrow[c, d]$; if $\phi$ is linear and if it does not create confusion, we will identify $\alpha$ and $\beta$. Under this identification, the restriction $\left.\alpha\right|_{[a, b]}$ of a path $\alpha:[0,1] \rightarrow X$ is a path itself with a path-homotopy class $\left[\left.\alpha\right|_{[a, b]}\right]$.

If $\alpha:[0,1] \rightarrow X$ is a path, then $\alpha^{-}(t)=\alpha(1-t)$ is the reverse path. If $\alpha_{1}, \alpha_{2}, \ldots, \alpha_{n}$ is a sequence of paths such that $\alpha_{j}(1)=\alpha_{j+1}(0)$ for each $j$, then $\prod_{j=1}^{n} \alpha_{j}=\alpha_{1} \cdot \alpha_{2} \cdot \cdots \cdot \alpha_{n}$ is the path defined as $\alpha_{j}$ on $\left[\frac{j-1}{n}, \frac{j}{n}\right]$. If $\alpha_{1}, \alpha_{2}, \alpha_{3}, \ldots$ is an infinite sequence of paths in $X$ such that $\alpha_{n}(1)=\alpha_{n+1}(0)$ for all $n \in \mathbb{N}$ and there is a point $x \in X$ such that every neighborhood of $x$ contains $\alpha_{n}([0,1])$ for all but finitely many $n$, then the infinite concatenation of this sequence is the path $\alpha=\prod_{n=1}^{\infty} \alpha_{n}=\left(\alpha_{1} \cdot\left(\alpha_{2} \cdot\left(\alpha_{3} \cdot(\cdots)\right)\right)\right)$ defined to be $\alpha_{n}$ on $\left[\frac{n-1}{n}, \frac{n}{n+1}\right]$ and $\alpha(1)=x$.

A path $\alpha:[a, b] \rightarrow X$ is reduced if $\alpha$ is constant or if whenever $a \leq s<t \leq b$ with $\alpha(s)=\alpha(t)$, the loop $\left.\alpha\right|_{[s, t]}$ is not null-homotopic. If $X$ is a one-dimensional metric space, then every path $\alpha:[0,1] \rightarrow X$ is path homotopic within the image of $\alpha$ to a reduced path, which is unique up to reparameterization [14.

For basic theory of linearly ordered sets, we refer to [23]. Let $\mathbf{n}, \omega$, and $\zeta$ denote the order types of the n-point set, the natural numbers, and $\mathbb{Z}$ respectively. If $(L, \leq)$ is a linearly ordered set, let $L^{*}$ denote $L$ with the reverse ordering $\leq^{*}$ where 
$b \leq^{*} a$ if and only if $a \leq b$. If $A, B \subseteq L$, then we write $A \leq B$ if $a \leq b$ for all $a \in A$ and $b \in B$.

Definition 2.1. Let $(L, \leq)$ be a linearly ordered set.

(1) $L$ is dense if $L$ has more than one point and if for each $x, y \in L$ with $x<y$, there exists $z \in L$ with $x<z<y$.

(2) $L$ is a scattered order if $L$ contains no dense suborders,

(3) A cut of $L$ is a pair $(A, B)$ of disjoint sets whose union is $L$ and $A<B$. The trivial cuts are the pairs where either $A=\emptyset$ or $B=\emptyset$. Let $\mathcal{C}(L)$ denote the set of cuts of $L$ with its natural linear ordering: $(A, B) \leq\left(A^{\prime}, B^{\prime}\right)$ if $A \subseteq A^{\prime}$.

Every countable linear order embeds as a suborder of the dense ordered set $\mathbb{Q}$.

Definition 2.2. A topological space $X$ is a scattered space if every non-empty subspace of $X$ contains an isolated point.

Every scattered order is a scattered space with the order topology. However, $\mathbb{Q} \times \mathbb{Z}$ with the lexicographical ordering is discrete but is not a scattered order since it contains a dense suborder. Generally, our use of the word "scattered" will be clear from context, however, if confusion is possible, we will clarify by specifically stating "scattered order" or "scattered space."

The following lemma is a combination of well-known results in linear order theory 23 and descriptive set theory 21] and will be used implicitly throughout the paper.

Lemma 2.3. For any countable linear order $L$, the following are equivalent:

(1) $L$ is a scattered order,

(2) $\mathcal{C}(L)$ embeds as a countable compact subset of $\mathbb{R}$,

(3) $\mathcal{C}(L)$ is a scattered space with the natural order topology.

Definition 2.4. If $K$ is a non-degenerate compact subset of $\mathbb{R}$, let $\mathcal{I}(K)$ denote the set of components of $[\min (K), \max (K)] \backslash K$ equipped with the ordering inherited from $\mathbb{R}$.

Note that $\mathcal{I}(K)$ is always countable and if $K$ is nowhere dense (for example, if $K$ is a scattered space), then $\mathcal{C}(\mathcal{I}(K)) \cong K$ as spaces. On the other hand, for any countable linear order $L$ and closed interval $[a, b]$ in $\mathbb{R}$, we may identify $\mathcal{C}(L)$ with a subspace of $[a, b]$ so that $a=\min (\mathcal{C}(L))$ and $b=\max (\mathcal{C}(L))$. Any such choice of embedding $\mathcal{C}(L) \subset[a, b]$ determines an order isomorphism $\mathcal{I}(\mathcal{C}(L)) \cong L$.

\section{CLOSURE OPERATORS ON SUBGROUPS OF FUNDAMENTAL GROUPS}

The following definitions are from [2] where closure operators of subgroups are introduced to characterize and compare local properties of fundamental groups.

Definition 3.1. Suppose $\left(\mathbb{T}, t_{0}\right)$ is a based space, $T \leq \pi_{1}\left(\mathbb{T}, t_{0}\right)$ is a subgroup, and $g \in \pi_{1}\left(\mathbb{T}, t_{0}\right)$. A subgroup $H \leq \pi_{1}\left(X, x_{0}\right)$ is $(T, g)$-closed if for every based map $f:\left(\mathbb{T}, t_{0}\right) \rightarrow\left(X, x_{0}\right)$ such that $f_{\#}(T) \leq H$, we also have $f_{\#}(g) \in H$. We refer to $(T, g)$ as a closure pair for $\left(\mathbb{T}, t_{0}\right)$.

The set of $(T, g)$-closed subgroups of $\pi_{1}\left(X, x_{0}\right)$ is closed under intersection and therefore forms a complete lattice. 
Definition 3.2. The $(T, g)$-closure of a subgroup $H \leq \pi_{1}\left(X, x_{0}\right)$ is

$$
\mathrm{Cl}_{T, g}(H)=\bigcap\left\{K \leq \pi_{1}\left(X, x_{0}\right) \mid K \text { is }(T, g) \text {-closed and } H \leq K\right\} .
$$

Lemma 3.3 (Closure Operator Properties of $\mathrm{Cl}_{T, g}$ ). [2, Section 2] Let $(T, g)$ be a closure pair. Then $\mathrm{Cl}_{T, g}(H)=H$ if and only if $H$ is $(T, g)$-closed. Moreover,

(1) $H \leq \mathrm{Cl}_{T, g}(H)$,

(2) $H \leq K$ implies $\mathrm{Cl}_{T, g}(H) \leq \mathrm{Cl}_{T, g}(K)$,

(3) $\mathrm{Cl}_{T, g}\left(\mathrm{Cl}_{T, g}(H)\right)=\mathrm{Cl}_{T, g}(H)$,

(4) if $f:\left(X, x_{0}\right) \rightarrow\left(Y, y_{0}\right)$ is a map, then $f_{\#}\left(\mathrm{Cl}_{T, g}(H)\right) \leq \mathrm{Cl}_{T, g}\left(f_{\#}(H)\right)$ in $\pi_{1}\left(Y, y_{0}\right)$.

The closure $\mathrm{Cl}_{T, g}(H)$ must contain the subgroup of $\pi_{1}\left(X, x_{0}\right)$ generated by $H$ and the set of elements $f_{\#}(g)$ for all maps $f:\left(T, t_{0}\right) \rightarrow\left(X, x_{0}\right)$ with $f_{\#}(T) \leq$ $H$. However, it is shown [2, Remark 3.13] that $\left\langle H \cup\left\{f_{\#}(g) \mid f:\left(T, t_{0}\right) \rightarrow\right.\right.$ $\left(X, x_{0}\right)$ with $\left.\left.f_{\#}(T) \leq H\right\}\right\rangle$ may not be $(T, g)$-closed and therefore may be a proper subgroup of $\mathrm{Cl}_{T, g}(H)$. In general, we must use the following inductive procedure to construct $\mathrm{Cl}_{T, g}(H)$ from $H$.

The Inductive Construction of Closure 3.4. Given a closure pair $(T, g)$ and subgroup $H \leq \pi_{1}\left(X, x_{0}\right)$, set $\mathrm{Cl}_{T, g}(H)_{\mathbf{0}}=H$ and suppose $\mathrm{Cl}_{T, g}(H)_{\lambda}$ is defined for all ordinals $\lambda<\kappa$. If $\kappa=\lambda+1$ is a successor, let $\mathrm{Cl}_{T, g}(H)_{\kappa}$ be the subgroup of $\pi_{1}\left(X, x_{0}\right)$ generated by $\mathrm{Cl}_{T, g}(H)_{\lambda}$ and the elements $f_{\#}(g)$ for all maps $f:\left(\mathbb{T}, t_{0}\right) \rightarrow$ $\left(X, x_{0}\right)$ satisfying $f_{\#}(T) \leq \mathrm{Cl}_{T, g}(H)_{\lambda}$. If $\kappa$ is a limit ordinal, let $\mathrm{Cl}_{T, g}(H)_{\kappa}=$ $\bigcup_{\lambda<\kappa} \mathrm{Cl}_{T, g}(H)_{\lambda}$, which is also a subgroup of $\pi_{1}\left(X, x_{0}\right)$.

Note that $\mathrm{Cl}_{T, g}(H)_{\kappa}$ is $(T, g)$-closed if and only if $\mathrm{Cl}_{T, g}(H)_{\kappa}=\mathrm{Cl}_{T, g}(H)_{\kappa+1}$. Hence, by basic cardinal considerations, there must be some smallest ordinal $\kappa_{0}$ such that $\mathrm{Cl}_{T, g}(H)_{\kappa}=\mathrm{Cl}_{T, g}(H)_{\kappa_{0}}$ for all $\kappa \geq \kappa_{0}$. By transfinite induction, every $(T, g)$-closed subgroup containing $H$ also contains $\mathrm{Cl}_{T, g}(H)_{\kappa}$ for each $\kappa$. Therefore, we have $\mathrm{Cl}_{T, g}(H)=\mathrm{Cl}_{T, g}(H)_{\kappa_{0}}=\bigcup_{\kappa} \mathrm{Cl}_{T, g}(H)_{\kappa}$.

Definition 3.5. The $(T, g)$-rank of an element $a \in \mathrm{Cl}_{T, g}(H)$, is the smallest ordinal $\kappa_{0}$ such that $a \in \mathrm{Cl}_{T, g}(H)_{\kappa_{0}}$.

We compare closure operators using the following remark.

Remark 3.6. 2, Proposition 2.3] If $(T, g)$ and $\left(T^{\prime}, g^{\prime}\right)$ are any closure pairs for a test space $\left(\mathbb{T}, t_{0}\right)$, then $g^{\prime} \in \mathrm{Cl}_{T, g}\left(T^{\prime}\right)$ if and only if $\mathrm{Cl}_{T^{\prime}, g^{\prime}}(H) \leq \mathrm{Cl}_{T, g}(H)$ for all spaces $\left(X, x_{0}\right)$ and subgroups $H \leq \pi_{1}\left(X, x_{0}\right)$.

Let $C_{n} \subseteq \mathbb{R}^{2}$ be the circle of radius $\frac{1}{n}$ centered at $\left(\frac{1}{n}, 0\right)$ and $\mathbb{H}=\bigcup_{n \in \mathbb{N}} C_{n}$ be the usual Hawaiian earring space with basepoint $b_{0}=(0,0)$. Let $\ell_{n}(t)=$ $\left(\frac{1}{n}(1-\cos (2 \pi t)),-\frac{1}{n} \sin (2 \pi t)\right)$ be the canonical counterclockwise loop traversing $C_{n}$ with homotopy class $a_{n}=\left[\ell_{n}\right]$. These elements freely generate the subgroup $F=\left\langle a_{n} \mid n \in \mathbb{N}\right\rangle \leq \pi_{1}\left(\mathbb{H}, b_{0}\right)$. Let $\ell_{\infty}$ denote the infinite concatenation $\prod_{n=1}^{\infty} \ell_{n}$ and $a_{\infty}=\left[\ell_{\infty}\right]$.

We will consider the closure operator $\mathrm{Cl}_{F, a_{\infty}}$ in the following section. Recall that this closure is constructed only using based maps $\left(\mathbb{H}, b_{0}\right) \rightarrow\left(X, x_{0}\right)$ and, therefore, only describes local homotopy structure at $x_{0}$.

\section{The SCATTERED EXTENSION OF A SUbGROUP}

Definition 4.1. Let $H \leq \pi_{1}\left(X, x_{0}\right)$ be a subgroup. A non-constant loop $\alpha$ : $[0,1] \rightarrow X$ based at $x_{0}$ is $H$-scattered if there exists a closed scattered space $K \subseteq$ 
$\alpha^{-1}\left(x_{0}\right)$ containing $\{0,1\}$ such that for every component $(a, b) \in \mathcal{I}(K)$, we have $\left[\left.\alpha\right|_{[a, b]}\right] \in H$. We call the set $K$ a set of $H$-cuts for $\alpha$. The scattered extension of $H$ is

$$
\operatorname{Sc}(H)=\left\{[\alpha] \in \pi_{1}\left(X, x_{0}\right) \mid \alpha \text { is } H \text {-scattered or constant }\right\} .
$$

Since the union of two closed scattered subspaces of $[0,1]$ is a scattered space, $\operatorname{Sc}(H)$ is a subgroup of $\pi_{1}\left(X, x_{0}\right)$ containing $H$.

Lemma 4.2. As an operator on subgroups of $\pi_{1}\left(X, x_{0}\right)$, Sc satisfies the following:

(1) $H \leq \mathrm{Sc}(H)$,

(2) $H \leq K \Rightarrow \mathrm{Sc}(H) \leq \mathrm{Sc}(K)$,

(3) $\mathrm{Sc}(H) \leq \mathrm{Sc}(\mathrm{Sc}(H))$,

(4) if $H \leq \pi_{1}\left(X, x_{0}\right)$ and $f:\left(X, x_{0}\right) \rightarrow\left(Y, y_{0}\right)$ is a map, then $f_{\#}(\operatorname{Sc}(H)) \leq$ $\operatorname{Sc}\left(f_{\#}(H)\right)$.

Proof. (1) and (2) are straightforward to verify and (3) follows from (1) and (2). To verify (4), suppose $[\alpha] \in \operatorname{Sc}(H)$ and $S \subseteq[0,1]$ is a set of $H$-cuts for representative $H$ scattered loop $\alpha$. For all $(a, b) \in \mathcal{I}(S)$, we have $\left[\left.\alpha\right|_{[a, b]}\right] \in H$ and, thus, $\left[\left.f \circ \alpha\right|_{[a, b]}\right] \in$ $f_{\#}(H)$. It follows that $S$ is a set of $f_{\#}(H)$-cuts for $f \circ \alpha$. Hence, $f_{\#}([\alpha]) \in$ $\operatorname{Sc}\left(f_{\#}(H)\right)$.

Remark 4.3. Compare the previous lemma with Lemma 3.3 , particularly the difference of property (3). We refrain from calling Sc a "closure" since, as an operator on subgroups, Sc behaves more like the first step $\mathrm{Cl}_{F, a_{\infty}}(-)_{1}$ in the inductive construction of $\mathrm{Cl}_{F, a_{\infty}}$. One should not expect $\mathrm{Sc}(\mathrm{Sc}(H))=\mathrm{Sc}(H)$ to hold in general.

For $H \leq \pi_{1}\left(X, x_{0}\right)$, each non-trivial element of $\mathrm{Sc}(H)$ is represented by a nonconstant loop $\alpha:[x, y] \rightarrow X$ based at $x_{0} \in X$ and a scattered set $S_{0} \subset \alpha^{-1}\left(x_{0}\right)$ of $H$-cuts for $\alpha$. For such a choice of representative $\alpha$ and $S_{0}$, we inductively define scattered subspaces $S_{\kappa} \subseteq S_{0}$ for each ordinal $\kappa$ so that $S_{\kappa+1}$ consists of $x, y$, and all non-isolated points of $S_{\kappa}$. If $\kappa$ is a limit ordinal, then $S_{\kappa}=\bigcap_{\lambda<\kappa} S_{\lambda}$. Since $S_{0}$ is a countable set (recall Lemma 2.3), there is a minimal countable ordinal $\kappa_{0}$ such that $S_{\kappa_{0}}=\{x, y\}$. We call $r k\left(\alpha, S_{0}\right)=\kappa_{0}$ the rank of $\alpha$ with respect to $S_{0}$ and note it's similarity to the Cantor-Bendixson rank of $S_{0}$ 21. Observe that if $(a, b) \in \mathcal{I}\left(S_{\kappa+1}\right)$, then $(a, b)$ is the union of the discrete (or empty) set $S_{\kappa} \cap(a, b)$ and the intervals in the set $\left\{(c, d) \in \mathcal{I}\left(S_{\kappa}\right) \mid(c, d) \subseteq(a, b)\right\}$, which has order type $\mathbf{n}, \omega, \omega^{*}$, or $\zeta$.

Lemma 4.4. Suppose $S_{0}$ is a set of $H$-cuts for $H$-scattered loop $\alpha:[x, y] \rightarrow X$ based at $x_{0}$. Suppose $(c, d) \in \mathcal{I}\left(S_{\kappa}\right)$ and $(a, b) \subseteq(c, d)$ where $a, b \in S_{\lambda}$ for $\lambda \leq \kappa$. Then $r k\left(\left.\alpha\right|_{[a, b]}, S_{0} \cap[a, b]\right) \leq \kappa$.

Proof. The sets $T_{0}=S_{0} \cap[a, b]$ and $U_{0}=S_{0} \cap[c, d]$ are sets of $H$-cuts for $\left.\alpha\right|_{[a, b]}$ and $\left.\alpha\right|_{[c, d]}$ respectively. Since $(c, d) \in \mathcal{I}\left(S_{\kappa}\right)$, it is clear that $r k\left(\left.\alpha\right|_{[c, d]}, T_{0}\right) \leq \kappa$. For any $\lambda^{\prime}$, every isolated point of $T_{\lambda^{\prime}} \cap(a, b)=S_{\lambda^{\prime}} \cap(a, b)$ is also an isolated point of $U_{\lambda^{\prime}} \cap(c, d)=S_{\lambda^{\prime}} \cap(c, d)$. Hence $r k\left(\left.\alpha\right|_{[a, b]}, T_{0}\right) \leq r k\left(\left.\alpha\right|_{[c, d]}, U_{0}\right) \leq \kappa$.

Corollary 4.5. Suppose $S_{0}$ is a set of $H$-cuts for $H$-scattered loop $\alpha:[x, y] \rightarrow X$. If $(c, d) \in \mathcal{I}\left(S_{\lambda}\right)$, then $T_{0}=S_{0} \cap[c, d]$ is a set of $H$-cuts for $\left.\alpha\right|_{[c, d]}$ and $r k\left(\left.\alpha\right|_{[c, d]}, T_{0}\right) \leq$ $\lambda$.

Lemma 4.6. If $H \leq \pi_{1}\left(X, x_{0}\right)$ and $\alpha:[x, y] \rightarrow X$ is a loop based at $x_{0}$ and $S \subseteq \alpha^{-1}\left(x_{0}\right)$ is a set of $\mathrm{Cl}_{F, a_{\infty}}(H)$-cuts for $\alpha$ such that $\mathcal{I}(S)$ has order type $\mathbf{n}, \omega$, $\omega^{*}$, or $\zeta$, then $[\alpha] \in \mathrm{Cl}_{F, a_{\infty}}(H)$. 
Proof. If $\mathcal{I}(S)$ has order type $\mathbf{n}$, then $[\alpha]$ factors as a finite product of elements of the subgroup $\mathrm{Cl}_{F, a_{\infty}}(H)$ and therefore lies in $\mathrm{Cl}_{F, a_{\infty}}(H)$. If $\mathcal{I}(S)$ can be ordered as

$$
\left(c_{1}, d_{1}\right)<\left(c_{2}, d_{2}\right)<\left(c_{3}, d_{3}\right)<\cdots
$$

define $f:\left(\mathbb{H}, b_{0}\right) \rightarrow\left(X, x_{0}\right)$ so that $\left.f \circ \ell_{n} \equiv \alpha\right|_{\left[c_{n}, d_{n}\right]}$. The continuity of $f$ follows from the continuity of $\alpha$. Since $f_{\#}\left(a_{n}\right)=\left[\left.\alpha\right|_{[c, d]}\right] \in \mathrm{Cl}_{F, a_{\infty}}(H)$ for all $n \in \mathbb{N}$, we have $[\alpha]=f_{\#}\left(a_{\infty}\right) \in \mathrm{Cl}_{F, a_{\infty}}(H)$. If $\mathcal{I}(S)$ has order type $\omega^{*}$, then $\mathcal{I}(\{x+y-t \mid$ $t \in S\}$ ) has order type $\omega$. The previous case applies to give $\left[\alpha^{-}\right] \in \mathrm{Cl}_{F, a_{\infty}}(H)$ and therefore $[\alpha] \in \mathrm{Cl}_{F, a_{\infty}}(H)$. If $\mathcal{I}(S)$ has order type of $\zeta$, fix any $t \in(x, y) \cap S$. Then $\mathcal{I}(S \cap[t, y])$ has order type $\omega$ and $\mathcal{I}(S \cap[x, t])$ has order type $\omega^{*}$. It follows that $\left[\left.\alpha\right|_{[t, y]}\right] \in \mathrm{Cl}_{F, a_{\infty}}(H)$ and $\left[\left.\alpha\right|_{[x, t]}\right] \in \mathrm{Cl}_{F, a_{\infty}}(H)$ by the first and second cases respectively. Thus $[\alpha]=\left[\left.\left.\alpha\right|_{[x, t]} \cdot \alpha\right|_{[t, y]}\right] \in \mathrm{Cl}_{F, a_{\infty}}(H)$.

Theorem 4.7. For any subgroup $H \leq \pi_{1}\left(X, x_{0}\right)$, we have $\operatorname{Sc}(H) \leq \mathrm{Cl}_{F, a_{\infty}}(H)$.

Proof. Every non-trivial element of $\operatorname{Sc}(H)$ is represented by a non-constant $H$ scattered loop $\alpha:[x, y] \rightarrow X$ based at $x_{0}$ that admits a set $S_{0}$ of $H$-cuts for $\alpha$. We show, by induction on $r k\left(\alpha, S_{0}\right)$, that $[\alpha] \in \mathrm{Cl}_{F, a_{\infty}}(H)$ for all such pairs $\left(\alpha, S_{0}\right)$. If $r k\left(\alpha, S_{0}\right)=0$, then $S_{0}=\alpha^{-1}\left(x_{0}\right)=\{x, y\}$ and it follows that $[\alpha] \in H \leq$ $\mathrm{Cl}_{F, a_{\infty}}(H)$. Suppose that for any $H$-scattered loop $\beta:[x, y] \rightarrow X$ based at $x_{0}$ and choice of $H$-cuts set $S_{0}$ with $r k\left(\beta, S_{0}\right)<\kappa$, we have $[\beta] \in \mathrm{Cl}_{F, a_{\infty}}(H)$. Consider $H$-scattered loop $\alpha$ based at $x_{0}$ with $H$-cut set $S_{0}$ such that $r k\left(\alpha, S_{0}\right)=\kappa$.

If $\kappa=\lambda+1$ is a successor ordinal, then $\mathcal{I}\left(S_{\lambda}\right)$ has order type $\mathbf{n}, \omega, \omega^{*}$, or $\zeta$. Fix $(c, d) \in \mathcal{I}\left(S_{\lambda}\right)$ and $T=S_{0} \cap[c, d]$, then $T$ is a set of $H$-cuts for $\left.\alpha\right|_{[c, d]}$ and, thus, $r k\left(\left.\alpha\right|_{[c, d]}, T\right) \leq \lambda$ by Corollary 4.5 . Since $r k\left(\left.\alpha\right|_{[c, d]}, T\right)<\kappa$, we have $\left[\alpha_{[c, d]}\right] \in \mathrm{Cl}_{F, a_{\infty}}(H)$ by our induction hypothesis. Since $\left[\alpha_{[c, d]}\right] \in \mathrm{Cl}_{F, a_{\infty}}(H)$ for all $(c, d) \in \mathcal{I}\left(S_{\lambda}\right)$, we have $[\alpha] \in \mathrm{Cl}_{F, a_{\infty}}(H)$ by Lemma 4.6

Recall that $\kappa$ is countable. So if $\kappa$ is a limit ordinal, we may find an $\omega$-sequence $0<\kappa_{1}<\kappa_{2}<\cdots<\kappa$ of countable ordinals converging to $\kappa$. Hence $\bigcap_{n \in \mathbb{N}} S_{\kappa_{n}}=$ $\{x, y\}$. Replacing $\left\{\kappa_{n}\right\}$ with a subsequence if necessary, we may find $\left(a_{n}, b_{n}\right) \in$ $\mathcal{I}\left(S_{\kappa_{n}}\right)$ so that

$$
\left(a_{1}, b_{1}\right) \subseteq\left(a_{2}, b_{2}\right) \subseteq \cdots \text { and } \bigcup_{n \in \mathbb{N}}\left(a_{n}, b_{n}\right)=(x, y)
$$

Note that it is possible for at most one of the sequences $\left\{a_{n}\right\}$ or $\left\{b_{n}\right\}$ to be eventually constant. By Lemma 4.5, we have $r k\left(\left.\alpha\right|_{\left[a_{n}, b_{n}\right]}, S_{0} \cap\left[a_{n}, b_{n}\right]\right) \leq \kappa_{n}<\kappa$. Therefore, $\left[\left.\alpha\right|_{\left[a_{n}, b_{n}\right]}\right] \in \mathrm{Cl}_{F, a_{\infty}}(H)$ for each $n \in \mathbb{N}$ by our induction hypothesis.

If $a_{n+1}<a_{n}<b_{n+1}$, then by Lemma 4.4 we have $r k\left(\left.\alpha\right|_{\left[a_{n+1}, a_{n}\right]}, S_{0} \cap\left[a_{n+1}, a_{n}\right]\right) \leq$ $\kappa_{n+1}<\kappa$ and, thus, $\left[\left.\alpha\right|_{\left[a_{n+1}, a_{n}\right]}\right] \in \mathrm{Cl}_{F, a_{\infty}}(H)$. Similarly, if $a_{n+1}<b_{n}<b_{n+1}$, then $\left[\left.\alpha\right|_{\left[b_{n}, b_{n+1}\right]}\right] \in \mathrm{Cl}_{F, a_{\infty}}(H)$. Now $A=\{x, y\} \cup\left\{a_{n} \mid n \in \mathbb{N}\right\} \cup\left\{b_{n} \mid n \in \mathbb{N}\right\}$ is a closed set in $A$ such that $\mathcal{I}(A)$ has order type $\omega, \omega^{*}$, or $\zeta$ and if $(c, d) \in \mathcal{I}(A)$, then $\left[\left.\alpha\right|_{[c, d]}\right] \in \mathrm{Cl}_{F, a_{\infty}}(H)$. By Lemma 4.6. we have $[\alpha] \in \mathrm{Cl}_{F, a_{\infty}}(H)$. This completes the induction.

Corollary 4.8. For any $H \leq \pi_{1}\left(X, x_{0}\right)$, we have

$$
\mathrm{Cl}_{F, a_{\infty}}(H)=\mathrm{Sc}\left(\mathrm{Cl}_{F, a_{\infty}}(H)\right)=\mathrm{Cl}_{F, a_{\infty}}(\mathrm{Sc}(H)) .
$$


Proof. Applying the operator properties of $\mathrm{Cl}_{F, a_{\infty}}$ (Lemma 3.3) and Sc (Lemma 4.2 and Theorem 4.7 the following sequence of inclusions proves the desired equalities.

$$
\begin{aligned}
\mathrm{Cl}_{F, a_{\infty}}(H) & \leq \mathrm{Sc}\left(\mathrm{Cl}_{F, a_{\infty}}(H)\right) \\
& \leq \mathrm{Sc}\left(\mathrm{Cl}_{F, a_{\infty}}(\mathrm{Sc}(H))\right) \\
& \leq \mathrm{Cl}_{F, a_{\infty}}\left(\mathrm{Cl}_{F, a_{\infty}}(\mathrm{Sc}(H))\right) \\
& =\mathrm{Cl}_{F, a_{\infty}}(\operatorname{Sc}(H)) \\
& \leq \mathrm{Cl}_{F, a_{\infty}}\left(\mathrm{Cl}_{F, a_{\infty}}(H)\right) \\
& =\mathrm{Cl}_{F, a_{\infty}}(H)
\end{aligned}
$$

Corollary 4.9. For any subgroup $H \leq \pi_{1}\left(X, x_{0}\right)$, the following are equivalent:

(1) $\operatorname{Sc}(H)$ is $\left(F, a_{\infty}\right)$-closed,

(2) $\mathrm{Sc}(H)=\mathrm{Cl}_{F, a_{\infty}}(H)$,

(3) $\mathrm{Sc}(H)=\operatorname{Sc}(\operatorname{Sc}(H))$.

Proof. By Theorem 4.7 and Corollary 4.8 the following inequality holds for all $H$ :

$$
\operatorname{Sc}(H) \leq \mathrm{Sc}(\mathrm{Sc}(H)) \leq \mathrm{Cl}_{F, a_{\infty}}(\mathrm{Sc}(H))=\mathrm{Cl}_{F, a_{\infty}}(H) .
$$

$(1) \Rightarrow(2) \Rightarrow(3)$ follows immediately. Suppose (3) holds, and $f:\left(\mathbb{H}, b_{0}\right) \rightarrow\left(X, x_{0}\right)$ is a map such that $f_{\#}(F) \leq \operatorname{Sc}(H)$. Then $\{1\} \cup\left\{\frac{n-1}{n} \mid n \in \mathbb{N}\right\}$ is a closed scattered set of $\operatorname{Sc}(H)$-cuts for $f \circ \ell_{\infty}$. Hence $f_{\#}\left(a_{\infty}\right)=\left[f \circ \ell_{\infty}\right] \in \operatorname{Sc}(\operatorname{Sc}(H))=\operatorname{Sc}(H)$. This proves $\operatorname{Sc}(H)$ is $\left(F, a_{\infty}\right)$-closed.

\section{The Homotopically Hausdorff Property and Scattered Products IN FUNDAMENTAL GROUPS}

Definition 5.1. 9, 19 We say a path-connected space $X$ is homotopically Hausdorff relative to a subgroup $H \leq \pi_{1}\left(X, x_{0}\right)$ if for every $x \in X$ and every path $\alpha:[0,1] \rightarrow X$ from $\alpha(0)=x_{0}$ to $\alpha(1)=x$, only the trivial right coset of $H^{\alpha}=\left[\alpha^{-}\right] H[\alpha]$ in $\pi_{1}(X, x)$ has arbitrarily small representatives, that is, if for every $g \in \pi_{1}(X, x) \backslash H^{\alpha}$, there is an open neighborhood $U$ of $x$ such that there is no loop $\delta:([0,1],\{0,1\}) \rightarrow(U, x)$ with $H^{\alpha} g=H^{\alpha}[\delta]$.

If $X$ is homotopically Hausdorff relative to the trivial subgroup $H=1$, we say $X$ is homotopically Hausdorff.

Remark 5.2. A space $X$ is homotopically Hausdorff if and only if for every point $x \in X$, there are no non-trivial elements of $\pi_{1}(X, x)$ that have a representative loop in every neighborhood of $x$.

Let $\mathbb{H}^{+}=\mathbb{H} \cup([-1,0] \times\{0\})$ be the Hawaiian earring with a "whisker" attached with basepoint $b_{0}^{+}=(-1,0)$ and where $\iota:[0,1] \rightarrow \mathbb{H}^{+}, \iota(t)=(t-1,0)$ is the inclusion of the whisker. Define $c_{n}=[\iota] a_{n}\left[\iota^{-}\right], c_{\infty}=[\iota] a_{\infty}\left[\iota^{-}\right]$, and $C=[\iota] F\left[\iota^{-}\right]$ in $\pi_{1}\left(\mathbb{H}^{+}, b_{0}^{+}\right)$. Equipped with Theorem 4.7, we provide a thorough study of the $\left(C, c_{\infty}\right)$-closure and explicitly compute the closures needed for our characterizations of the homotopically Hausdorff property.

Proposition 5.3. For any $H \leq \pi_{1}\left(X, x_{0}\right)$, we have $\mathrm{Cl}_{F, a_{\infty}}(H) \leq \mathrm{Cl}_{C, c_{\infty}}(H)$. 
Proof. The retraction $f: \mathbb{H}^{+} \rightarrow \mathbb{H}$ collapsing the whisker to a point satisfies $f_{\#}(C)=F$ and $f_{\#}\left(c_{\infty}\right)=a_{\infty}$. Hence, $a_{\infty} \in \mathrm{Cl}_{C, c_{\infty}}(F)$ and we may apply Remark 3.6 .

Corollary 5.4. For any $H \leq \pi_{1}\left(X, x_{0}\right)$, we have

$$
\mathrm{Cl}_{C, c_{\infty}}(H)=\mathrm{Sc}\left(\mathrm{Cl}_{C, c_{\infty}}(H)\right)=\mathrm{Cl}_{C, c_{\infty}}(\mathrm{Sc}(H)) .
$$

Proof. For any $H \leq \pi_{1}\left(X, x_{0}\right)$, Theorem 4.7 and Proposition 5.3 give the inclusion $\mathrm{Sc}(H) \leq \mathrm{Cl}_{C, c_{\infty}}(H)$. Hence, the analogous sequence of inclusions used in the proof of Corollary 4.8 applies.

Corollary 5.5. For any $H \leq \pi_{1}\left(X, x_{0}\right)$, we have $\operatorname{Sc}(H)=\mathrm{Cl}_{C, c_{\infty}}(H)$ if and only if $\operatorname{Sc}(H)$ is $\left(C, c_{\infty}\right)$-closed.

Definition 5.6. For a space $X$ and subset $A \subseteq X$, let

$$
\begin{aligned}
& F(X, A)=\left\{[\alpha] \in \pi_{1}\left(X, x_{0}\right) \mid \alpha^{-1}(A) \text { is finite or } \alpha \text { is constant }\right\} \\
& \operatorname{Sc}(X, A)=\left\{[\alpha] \in \pi_{1}\left(X, x_{0}\right) \mid \alpha^{-1}(A) \text { is a scattered space or } \alpha \text { is constant }\right\} .
\end{aligned}
$$

Note that $F(X, A)$ and $\operatorname{Sc}(X, A)$ are subgroups of $\pi_{1}\left(X, x_{0}\right)$ such that $\operatorname{Sc}(X, A) \leq$ $\operatorname{Sc}(F(X, A))$. In fact, since the closed scattered subspaces of $\mathbb{R}$ are precisely the closed countable subsets of $\mathbb{R}, \operatorname{Sc}(X, A)$ is precisely the subgroup of countable cutpoints $C C P\left(X, A, x_{0}\right)$ in [2, Example 3.13].

Example 5.7. As a special case, we call the group $S c=\operatorname{Sc}\left(\mathbb{H},\left\{b_{0}\right\}\right) \leq \pi_{1}\left(\mathbb{H}, b_{0}\right)$ the subgroup of scattered words. It is known that $S c$ is algebraically free on uncountably many generators (See [6] or [13]).

Proposition 5.8. [2, Proposition 3.14] If $X$ is a one-dimensional metric space and $A \subseteq X$ is closed, then $\operatorname{Sc}(X, A)$ is $\left(C, c_{\infty}\right)$-closed.

Lemma 5.9. If $X$ is a one-dimensional metric space and $A \subseteq X$ is closed, then $\operatorname{Sc}(F(X, A))=\operatorname{Sc}(X, A)$.

Proof. The inclusion $\operatorname{Sc}(X, A) \leq \operatorname{Sc}(F(X, A))$ is clear. Suppose $1 \neq[\alpha] \in \operatorname{Sc}(F(X, A))$ for $F(X, A)$-scattered loop $\alpha$. Take $K \subseteq \alpha^{-1}\left(x_{0}\right)$ to be a set of $F(X, A)$-cuts for loop $\alpha:[0,1] \rightarrow X$. We define a new path $\beta:[0,1] \rightarrow X$ as follows: fix $(c, d) \in \mathcal{I}(K)$. Then $\left.\alpha\right|_{[c, d]}$ is path-homotopic to a path $\gamma:[c, d] \rightarrow X$ such that $\gamma^{-1}(A)$ is finite. Since reduction of any path only occurs within the image of that path, the preimage of $A$ under the reduced representative of $\gamma$ is also finite. However, this reduced representative is unique to the homotopy class and is therefore also the reduced representative of $\left.\alpha\right|_{[c, d]}$. Hence, we define $\beta$ so that $\left.\beta\right|_{K}=\left.\alpha\right|_{K}$ and $\left.\beta\right|_{[c, d]}$ is the reduced representative of $\left.\alpha\right|_{[c, d]}$ for all $(c, d) \in \mathcal{I}(K)$. Continuity of $\beta$ at a point $k \in K$ follows from the continuity of $\alpha$ at $k$ and the fact that $\beta([c, d]) \subset \alpha([c, d])$ for all $(c, d) \in \mathcal{I}(K)$. Since, to construct $\beta$, we have only performed reductions of subpaths of $\alpha$, it is clear that $\alpha$ and $\beta$ have the same reduced representative and are therefore homotopic. Notice that $\beta^{-1}(A)$ is closed and is the union of $K$ and at most finitely many isolated points in each interval $(c, d) \in \mathcal{I}(K)$. Hence, since $K$ is scattered, $\beta^{-1}(A)$ is scattered. Therefore, $[\alpha]=[\beta] \in \operatorname{Sc}(X, A)$.

Theorem 5.10. If $X$ is a one-dimensional metric space and $A \subseteq X$ is closed, then $\mathrm{Cl}_{C, c_{\infty}}(F(X, A))=\mathrm{Sc}(X, A)$. 
Proof. Together, Proposition 5.8 and Lemma 5.9 give that $\operatorname{Sc}(X, A)=\operatorname{Sc}(F(X, A))$ is $\left(C, c_{\infty}\right)$-closed. Applying Corollary 5.5 to $H=F(X, A)$ gives $\operatorname{Sc}(F(X, A))=$ $\mathrm{Cl}_{C, c_{\infty}}(F(X, A))$. Hence, $\mathrm{Cl}_{C, c_{\infty}}(F(X, A))=\mathrm{Sc}(X, A)$.

Corollary 5.11. $\mathrm{Cl}_{C, c_{\infty}}(F)=\mathrm{Sc}(F)=S c$ and $\mathrm{Cl}_{C, c_{\infty}}(C)=[\iota] S c\left[\iota^{-}\right]$.

Proof. Observe that $F=F\left(\mathbb{H},\left\{b_{0}\right\}\right), S c=\mathrm{Sc}\left(\mathbb{H},\left\{b_{0}\right\}\right), C=F\left(\mathbb{H}^{+},\left\{b_{0}\right\}\right) \leq$ $\pi_{1}\left(\mathbb{H}^{+}, b_{0}^{+}\right)$, and $\operatorname{Sc}\left(\mathbb{H}^{+},\left\{b_{0}\right\}\right)=[\iota] \mathrm{Sc}\left(\mathbb{H},\left\{b_{0}\right\}\right)\left[\iota^{-}\right]=[\iota] S c\left[\iota^{-}\right]$. Applying Theorem 5.10 gives both equalities.

We complete this section by proving the equivalence $(1) \Leftrightarrow(2) \Leftrightarrow(5)$ in Theorem 1.2. Let $\ell_{\geq m}$ denote the infinite concatenation $\prod_{n=m}^{\infty} \ell_{n}$ for $m \in \mathbb{N}$.

Theorem 5.12. For any space $X$, the following are equivalent:

(1) the trivial subgroup of $\pi_{1}\left(X, x_{0}\right)$ is $\left(C, c_{\infty}\right)$-closed,

(2) for any maps $f, g:\left(\mathbb{H}, b_{0}\right) \rightarrow(X, x)$ such that $f_{\#}\left(a_{n}\right)=g_{\#}\left(a_{n}\right)$ for all $n \in \mathbb{N}$, we have $f_{\#}\left(a_{\infty}\right)=g_{\#}\left(a_{\infty}\right)$,

(3) for any maps $f, g:\left(\mathbb{H}, b_{0}\right) \rightarrow(X, x)$ such that $f_{\#}\left(a_{n}\right)=g_{\#}\left(a_{n}\right)$ for all $n \in \mathbb{N}$, we have $\left.f_{\#}\right|_{S c}=\left.g_{\#}\right|_{S c}$, i.e. $f_{\#}$ and $g_{\#}$ agree on the subgroup of scattered words.

If $X$ is first countable, these are also equivalent to the homotopically Hausdorff property.

Proof. (1) $\Rightarrow(2)$ Suppose $1 \leq \pi_{1}\left(X, x_{0}\right)$ is $\left(C, c_{\infty}\right)$-closed and that $f, g:\left(\mathbb{H}, b_{0}\right) \rightarrow$ $(X, x)$ are maps satisfying $f_{\#}\left(a_{n}\right)=g_{\#}\left(a_{n}\right)$ for all $n \in \mathbb{N}$. Define $\gamma_{n}=\left(f \circ \ell_{\geq n}\right)^{-}$. $\left(g \circ \ell_{\geq n}\right), n \in \mathbb{N}$. For all $n$, we have $\left[\left(f \circ \ell_{n}\right)^{-} \cdot\left(g \circ \ell_{n}\right)\right]=1$ and, thus, $\gamma_{n} \simeq \gamma_{n+1}$. Let $\alpha:[0,1] \rightarrow X$ be any path from $x_{0}$ to $x$ and define $k:\left(\mathbb{H}^{+}, b_{0}^{+}\right) \rightarrow\left(X, x_{0}\right)$ by $k \circ \iota=\alpha$ and $k \circ \ell_{n}=\gamma_{n} \cdot \gamma_{n+1}^{-}$. Then $k_{\#}(C)=1$ and a swindle yields $k_{\#}\left(c_{\infty}\right)=[\alpha]\left[\gamma_{1}\right]\left[\alpha^{-}\right]$. Since the trivial subgroup is assumed to be $\left(C, c_{\infty}\right)$-closed, we must have $\left[\gamma_{1}\right]=f_{\#}\left(a_{\infty}\right)^{-1} g_{\#}\left(a_{\infty}\right)=1$ in $\pi_{1}(X, x)$. Thus $f_{\#}\left(a_{\infty}\right)=g_{\#}\left(a_{\infty}\right)$.

$(2) \Rightarrow(3)$ Suppose $(2)$ holds and $f, g:\left(\mathbb{H}, b_{0}\right) \rightarrow(X, x)$ are maps satisfying $\left.f_{\#}\right|_{F}=\left.g_{\#}\right|_{F}$. By Corollary 5.11, we have $S c=\mathrm{Cl}_{C, c_{\infty}}(F)$ so we show that $f_{\#}$ and $g_{\#}$ agree on every element of $\mathrm{Cl}_{C, c_{\infty}}(F)$. We proceed by induction on the $\left(F, a_{\infty}\right)$-rank of the elements of $\mathrm{Cl}_{F, a_{\infty}}(F)$. By assumption, $f_{\#}$ and $g_{\#}$ agree on the elements of $\mathrm{Cl}_{F, a_{\infty}}(F)_{0}=F$. Suppose $f_{\#}(a)=g_{\#}(a)$ for all elements $a \in$ $\mathrm{Cl}_{F, a_{\infty}}(F)$ with $\left(F, a_{\infty}\right)$-rank $<\kappa$. Suppose $a \in \mathrm{Cl}_{F, a_{\infty}}(F)$ has $\left(F, a_{\infty}\right)$-rank $\kappa$. Since $\kappa$ must necessarily be a successor ordinal, we have $\kappa=\lambda+1$ for some ordinal $\lambda$. Then $a=\prod_{i=1}^{m} b_{i}$ where either $b_{i}$ has $\left(F, a_{\infty}\right)$-rank $\leq \lambda$ or $b_{i}=k_{\#}\left(a_{\infty}\right)$ for $k:\left(\mathbb{H}, b_{0}\right) \rightarrow\left(\mathbb{H}, b_{0}\right)$ with $k_{\#}(F) \leq \mathrm{Cl}_{F, a_{\infty}}(F)_{\lambda}$. In the first case, $f_{\#}$ and $g_{\#}$ agree on $b_{i}$ by the induction hypothesis so we focus on the case where $b_{i}=k_{\#}\left(a_{\infty}\right)$. For each $n \in \mathbb{N}$, we have $k_{\#}\left(a_{n}\right) \in \mathrm{Cl}_{F, a_{\infty}}(F)_{\lambda}$ and, thus, $f_{\#}\left(k_{\#}\left(a_{n}\right)\right)=g_{\#}\left(k_{\#}\left(a_{n}\right)\right)$ by the induction hypothesis. Since $(2)$ holds and $f \circ k, g \circ k$ are maps $\mathbb{H} \rightarrow \mathbb{H}$ whose induced homomorphisms agree on $F$, we have $f_{\#}\left(b_{i}\right)=f_{\#}\left(k_{\#}\left(a_{\infty}\right)\right)=$ $g_{\#}\left(k_{\#}\left(a_{\infty}\right)\right)=g_{\#}\left(b_{i}\right)$. Since $f_{\#}$ and $g_{\#}$ agree on the factors of $a$, we have $f_{\#}(a)=$ $g_{\#}(a)$, completing the induction.

$(3) \Rightarrow(1)$ Suppose $1 \leq \pi_{1}\left(X, x_{0}\right)$ is not $\left(C, c_{\infty}\right)$-closed. Then there exists, $k:\left(\mathbb{H}^{+}, b_{0}^{+}\right) \rightarrow\left(X, x_{0}\right)$ such that $k_{\#}\left(c_{n}\right)=1$ for all $n \in \mathbb{N}$ and $k_{\#}\left(c_{\infty}\right) \neq 1$. Let $x=k\left(b_{0}\right), f=\left.k\right|_{\mathbb{H}}$, and $g: \mathbb{H} \rightarrow X$ be the constant map at $x$. Then $f_{\#}\left(a_{n}\right)=1=g_{\#}\left(a_{n}\right)$ in $\pi_{1}(X, x)$ for all $n$. Using the basepoint change isomorphism $\phi: \pi_{1}\left(X, x_{0}\right) \rightarrow \pi_{1}(X, x), \phi([\alpha])=\left[(k \circ \iota)^{-} \cdot \alpha \cdot(k \circ \iota)\right]$, we see that $f_{\#}\left(a_{\infty}\right)=$ $\phi\left(k_{\#}\left(c_{\infty}\right)\right) \neq 1$. Hence, $f_{\#}$ and $g_{\#}$ do not agree on $a_{\infty} \in \mathrm{Cl}_{F, a_{\infty}}(F)=S c$. 
The last statement of the theorem is a special case of Theorem 3.6 in [2]. We include a proof for the sake of completeness.

(hom. Hausdorff) $\Rightarrow(1)$ If $1 \leq \pi_{1}\left(X, x_{0}\right)$ is not $\left(C, c_{\infty}\right)$-closed, then there exists a map $f:\left(\mathbb{H}^{+}, b_{0}^{+}\right) \rightarrow\left(X, x_{0}\right)$ such that $f_{\#}(C)=1$ and $f_{\#}\left(c_{\infty}\right) \neq 1$. Setting $\alpha=f \circ \iota$, we have $f_{\#}\left(a_{n}\right)=\left[\alpha^{-}\right] f_{\#}\left(c_{n}\right)[\alpha]=1$ for all $n \in \mathbb{N}$ and $g=f_{\#}\left(a_{\infty}\right) \neq 1$. Let $U$ be any open neighborhood of $x=f\left(b_{0}\right)$. By the conintuity of $f$, there exists an $m \in \mathbb{N}$ such that $\delta=f \circ \ell_{\geq m}$ has image in $U$. Then $g=f_{\#}\left(a_{1} a_{2} \ldots a_{m-1}\right)[\delta]=[\delta]$, showing that $X$ is not homotopically Hausdorff.

(1) and $X$ first countable $\Rightarrow$ (hom. Hausdorff) Suppose $X$ is first countable and is not homotopically Hausdorff. Then there exists $1 \neq g \in \pi_{1}\left(X, x_{0}\right)$ and a path $\alpha$ starting at $x_{0}$ such that for every neighborhood $U$ of $x=\alpha(1)$, there exists a loop $\delta$ in $U$ based at $x$ such that $g=[\delta]$. Let $U_{1} \supseteq U_{2} \supseteq U_{3} \supseteq \cdots$ be a neighborhood base at $x$. By assumption, there exists loops $\delta_{n}$ in $U_{n}$ based at $x$ such that $g=\left[\delta_{n}\right]$. Define $f: \mathbb{H}^{+} \rightarrow X$ by $f \circ \iota=\alpha$ and $f \circ \ell_{n}=\delta_{n} \cdot \delta_{n+1}^{-}$. Since the basis $\left\{U_{n} \mid n \in \mathbb{N}\right\}$ is nested, $f$ is continuous at $b_{0}$. Note that $f_{\#}\left(c_{n}\right)=\left[\alpha \cdot \delta_{n} \cdot \alpha^{-}\right]\left[\alpha \cdot \delta_{n+1} \cdot \alpha^{-}\right]^{-1}=1$ for all $n \in \mathbb{N}$ and, thus, $f_{\#}(C)=1$. However, applying a swindle gives $f_{\#}\left(c_{\infty}\right)=$ $\left[\alpha \cdot \delta_{1}\right]\left[\prod_{n=2}^{\infty}\left(\delta_{n}^{-} \cdot \delta_{n}\right)\right]\left[\alpha^{-}\right]=\left[\alpha \cdot \delta_{1} \cdot \alpha^{-}\right] \neq 1$. We conclude that the trivial subgroup $1 \leq \pi_{1}\left(X, x_{0}\right)$ is not $\left(C, c_{\infty}\right)$-closed.

Example 5.13. Let $\mathbb{H} \mathbb{A} \subseteq \mathbb{R}^{3}$ be the harmonic archipelago space [7] where $\mathbb{R}^{2} \times$ $\{0\} \cap \mathbb{H} \mathbb{A}=\mathbb{H} \times\{0\}$. Then infinite $\pi_{1}$-products are not well-defined in $\pi_{1}\left(\mathbb{H} \mathbb{A}, b_{0}\right)$. Note that $1 \neq\left[\ell_{1}\right]=\left[\ell_{2}\right]=\cdots$ in $\pi_{1}\left(\mathbb{H} \mathbb{A}, b_{0}\right)$ and define $\gamma_{n}=\ell_{n} \cdot \ell_{n+1}^{-}$. If $f: \mathbb{H} \rightarrow$ $\mathbb{H} \mathbb{A}$ is the constant map at $b_{0}$ and $g: \mathbb{H} \rightarrow \mathbb{H} \mathbb{A}$ satisfies $g \circ \ell_{n}=\ell_{n} \cdot \ell_{n+1}^{-}$, then we have $f_{\#}\left(a_{n}\right)=g_{\#}\left(a_{n}\right)$ for all $n \in \mathbb{N}$. However, $f_{\#}\left(a_{\infty}\right)=1 \neq\left[\ell_{1}\right]=g_{\#}\left(a_{\infty}\right)$. Hence, $\prod_{n=1}^{\infty}\left[\gamma_{n}\right]$ does not have a well-defined meaning in $\pi_{1}\left(\mathbb{H} \mathbb{A}, b_{0}\right)$.

In general, if a space fails to have the homotopically Hausdorff property, one is guaranteed the existence of a net of loops based at a point $x$, within a single nontrivial homotopy class, that converges to the constant loop at $x$ in the compact-open topology. However, to form topologically represented infinite products in $\pi_{1}$, one must have a sequence of such loops. Without assuming first countability, it may not be possible to replace a net with a cofinal subsequence. The following higher-ordinal analogue of the harmonic archipelago illustrates why it is necessary to assume first countability if we wish to include the homotopically Hausdorff property in the statements of Theorem 1.2 and Theorem 5.12

Example 5.14. Let $X$ be the reduced suspension of the first uncountable compact ordinal $\omega_{1}+1=\omega_{1} \cup\left\{\omega_{1}\right\}$ with canonical basepoint $x_{0}$. For $\lambda<\omega_{1}$, let $C_{\lambda}$ be the circle, which is the image of $\{\lambda\} \times[0,1]$ in $X$, and let $\gamma_{\lambda}$ be the canonical loop traversing $C_{\lambda}$. Since $C_{\lambda}$ is a retract of $X$, each $\gamma_{\lambda}$ is homotopically non-trivial. Let $Y$ be the space obtained by attaching a 2-cell to $X$ along the loop $\gamma_{\mu} \cdot \gamma_{\lambda}^{-}$for all ordered pairs $\mu<\lambda<\omega_{1}$. Since no sequence of countable ordinals converges to $\omega_{1}$, there is no sequentially compact subspace of $X$ that contains $C_{\lambda}$ for infinitely many $\lambda$. Therefore, infinite concatenations of sequences of non-trivial loops can be formed in neither $X$ nor $Y$. It follows that $\pi_{1}\left(X, x_{0}\right)$ is free on the uncountable set $\left\{\left[\gamma_{\lambda}\right] \mid \lambda<\omega_{1}\right\}$, the group $\pi_{1}\left(Y, y_{0}\right)$ is infinite cyclic (generated by the homotopy class containing $\left.\left\{\gamma_{\lambda} \mid \lambda<\omega_{1}\right\}\right)$, and the trivial subgroup is $\left(C, c_{\infty}\right)$-closed in both $\pi_{1}\left(X, x_{0}\right)$ and $\pi_{1}\left(Y, x_{0}\right)$. However, since every neighborhood of $x_{0}$ in $Y$ contains $C_{\lambda}$ for some $\lambda<\omega_{1}, Y$ is not homotopically Hausdorff. 


\section{SCATTERED PRODUCTS IN FUNDAMENTAL GROUPOIDS}

We now introduce a set of closure pairs, which will be useful for transitioning from infinite products in fundamental groups to infinite products in fundamental groupoids.

Let $A$ be a closed proper subset of $[0,1]$ containing $\{0,1\}$. For each $I=(a, b) \in$ $\mathcal{I}(A)$, let $C_{I}=\left\{(x, y) \in \mathbb{R}^{2} \mid y \geq 0,\left(x-\frac{a+b}{2}\right)^{2}+y^{2}=\left(\frac{b-a}{2}\right)^{2}\right\}$ be the semicircle whose boundary is $\{(a, 0),(b, 0)\}$. Let $\mathbb{W}_{A}=B \cup \bigcup_{I \in \mathcal{I}(A)} C_{I}$ with basepoint $w_{0}=$ $(0,0)$ where $B=[0,1] \times\{0\}$ is the base-arc.

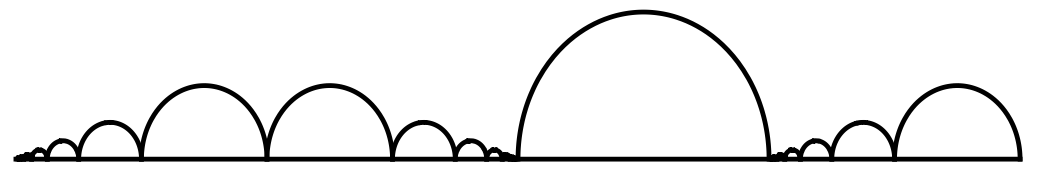

Figure 1. An example of the space $\mathbb{W}_{A}$ where $A$ is scattered and $\mathcal{I}(A)$ has order type $\zeta+\mathbf{1}+\omega^{*}$.

For $I=(a, b) \in \mathcal{I}(A)$, let $\lambda_{I}:[0,1] \rightarrow B$ be the path $\lambda_{I}(t)=(b t+a(1-t), 0)$ and $v_{I}:[0,1] \rightarrow C_{I}$ be the path so that if $r: \mathbb{W}_{A} \rightarrow B$ is the projection onto the $\mathrm{x}$-axis, then $r \circ v_{I}=\lambda_{I}$. Let $\lambda(t)=(t, 0)$ and $v:[0,1] \rightarrow(A \times\{0\}) \cup \bigcup_{I \in \mathcal{I}(A)} C_{I}$ be the path such that $r \circ v=\lambda$. Hence, $\lambda$ and $v$ are paths in $\mathbb{W}_{A}$ from $w_{0}$ to $(1,0)$ such that $\lambda$ is the path along the base-arc and $v$ is the path through the circles $C_{I}$.

Let $W_{A}$ be the subgroup of $\pi_{1}\left(\mathbb{W}_{A}, w_{0}\right)$ generated by the elements $w_{I}=\left[v_{[0, b]}\right.$. $\left.\lambda_{[a, b]}^{-} \cdot v_{[0, a]}^{-}\right]$for $I=(a, b) \in \mathcal{I}(A)$ and let $w_{A, \infty}=\left[v \cdot \lambda^{-}\right]$. We consider the closure pair $\left(W_{A}, w_{A, \infty}\right)$ for the test space $\left(\mathbb{W}_{A}, w_{0}\right)$.

Remark 6.1. If $A$ is finite, then $\mathrm{Cl}_{W_{A}, w_{A, \infty}}$ is the identity closure operator. If $\mathcal{C}$ is the ternary Cantor set, then the operator $\mathrm{Cl}_{W_{\mathcal{C}}, w_{\mathcal{C}, \infty}}$ is the closure pair used in [2, Section 7] to characterize the well-definedness of transfinite $\Pi_{1}$-products.

Lemma 6.2. Let $A=\left\{\frac{n-1}{n} \mid n \in \mathbb{N}\right\} \cup\{1\}$. Then for any subgroup $H \leq \pi_{1}\left(X, x_{0}\right)$, $\mathrm{Cl}_{C, c_{\infty}}(H)=\mathrm{Cl}_{W_{A}, w_{A, \infty}}(H)$.

Proof. By Remark 3.6 it suffices to show $w_{A, \infty} \in \mathrm{Cl}_{C, c_{\infty}}\left(W_{A}\right)$ and $c_{\infty} \in \mathrm{Cl}_{W_{A}, w_{A, \infty}}(C)$.

Let $t_{n}=\frac{n-1}{n}$ so that the components of $[0,1] \backslash A$ are $I_{n}=\left(t_{n}, t_{n+1}\right), n \in \mathbb{N}$. Set $v_{n}:=v_{I_{n}}, \lambda_{n}:=\lambda_{I_{n}}$, and $w_{n}:=w_{I_{n}}$. Let $v_{\geq n}=\prod_{k=n}^{\infty} v_{k}$ and $\lambda_{\geq n}(t)=$ $\lambda\left(t+(1-t) t_{n}\right)$ be the path along the base-arc from $\left(t_{n}, 0\right)$ to $(1,0)$.

Define $f:\left(\mathbb{H}^{+}, b_{0}^{+}\right) \rightarrow\left(\mathbb{W}_{A}, w_{0}\right)$ so that $f \circ \iota=v$ and $f \circ \ell_{n} \equiv v_{\geq n}^{-} \cdot \lambda_{n} \cdot v_{\geq n+1}$. Then $f_{\#}\left(c_{n}\right)=\left[v \cdot v_{\geq n}^{-} \cdot \lambda_{n} \cdot v_{\geq n+1} \cdot v^{-}\right]=\left[v_{\left[0, t_{n}\right]} \cdot \lambda_{n} \cdot v_{\left[0, t_{n+1}\right]}^{-}\right]=\left(w_{n}\right)^{-1} \in W_{A}$ and $f_{\#}\left(c_{\infty}\right)=\left[v \cdot\left(\prod_{n=1}^{\infty} v_{\geq n}^{-} \cdot \lambda_{n} \cdot v_{\geq n+1}\right) \cdot v^{-}\right]=\left[\lambda \cdot v^{-}\right]=w_{A, \infty}^{-1}$. This implies $w_{A, \infty}^{-1} \in \mathrm{Cl}_{C, c_{\infty}}\left(W_{A}\right)$, hence $w_{A, \infty} \in \mathrm{Cl}_{C, c_{\infty}}\left(W_{A}\right)$.

Define $g:\left(\mathbb{W}_{A}, w_{0}\right) \rightarrow\left(\mathbb{H}^{+}, b_{0}^{+}\right)$so that $g \circ v_{1} \equiv g \circ \lambda_{1} \equiv \iota$ and $g \circ v_{n}$ is constant at $b_{0}$ for $n \geq 2$ and $g \circ \lambda_{n}=\ell_{n-1}$ for $n \geq 2$. Then $g_{\#}\left(w_{1}\right)=1, g_{\#}\left(w_{n}\right)=$ $\left[\iota \cdot\left(g \circ \lambda_{n}^{-}\right) \cdot \iota^{-}\right]=c_{n-1}^{-1} \in C$ for all $n \geq 2$, and $g_{\#}\left(w_{A, \infty}\right)=\left[\iota \cdot\left(g \circ \lambda_{n \geq 2}^{-}\right) \cdot \iota^{-}\right]=c_{\infty}^{-1}$. This implies $c_{\infty}^{-1} \in \mathrm{Cl}_{W_{A}, w_{A}, \infty}(C)$, hence $c_{\infty} \in \mathrm{Cl}_{W_{A}, w_{A}, \infty}(C)$. 
Definition 6.3. Let $H \leq \pi_{1}\left(X, x_{0}\right)$ be a subgroup. The scattered path-product closure of $H$, denoted $\mathrm{Cl}_{\text {spp }}(H)$, is the subgroup of $\pi_{1}\left(X, x_{0}\right)$ generated by the union $\bigcup_{A} \mathrm{Cl}_{W_{A}, w_{A, \infty}}(H)$ taken over all closed scattered sets $A \subset[0,1]$ containing $\{0,1\}$. We say that $X$ has well-defined scattered $\Pi_{1}$-products relative to $H$ if $H=\mathrm{Cl}_{s p p}(H)$.

When $H=1$, the condition $\mathrm{Cl}_{\text {spp }}(1)=1$ in $\pi_{1}\left(X, x_{0}\right)$ is clearly equivalent to well-definedness of scattered $\Pi_{1}$-products in $X$ (recall (4) of Definition 1.1). The following lemma is an immediate consequence of the definition of $\mathrm{Cl}_{s p p}$ and the closure properties of $\mathrm{Cl}_{W_{A}, w_{A, \infty}}$ (recall Lemma 3.3).

Lemma 6.4. $\mathrm{Cl}_{\text {spp }}(H)=H$ if and only if $H$ is $\left(W_{A}, w_{A, \infty}\right)$-closed for every closed scattered set $A \subset[0,1]$ containing $\{0,1\}$. In particular, the operator $\mathrm{Cl}_{\text {spp }}$ has all of the properties appearing in Lemma 3.3 .

Remark 6.5. If $N \unlhd \pi_{1}\left(X, x_{0}\right)$ is a normal subgroup, then $\mathrm{Cl}_{W_{A}, w_{A, \infty}}(N)$ and $\mathrm{Cl}_{W_{A^{\prime}}, w_{A^{\prime}, \infty}}(N)$ are contained in the normal subgroup $\mathrm{Cl}_{W_{B}, w_{B, \infty}}(N)$ where $B=$ $\{0\} \cup\left\{\frac{t+1}{3} \mid t \in A\right\} \cup\left\{\frac{t+2}{3} \mid t \in A^{\prime}\right\}$ (See 2, Lemma 2.9]). Hence, $\mathrm{Cl}_{\text {spp }}(N)=$ $\bigcup_{A} \mathrm{Cl}_{W_{A}, w_{A, \infty}}(N)$ is a normal subgroup.

Theorem 6.6. A space $X$ has well-defined scattered $\Pi_{1}$-products relative to $H$ if and only if $H$ is $\left(C, c_{\infty}\right)$-closed. Moreover, if $X$ is first countable, these properties are equivalent to the homotopically Hausdorff relative to $H$ property.

Proof. We focus on the first statement since [2, Theorem 3.6] states that homotopically Hausdorff rel. $H \Rightarrow H$ is $\left(C, c_{\infty}\right)$-closed and that the converse holds when $X$ is first countable.

Suppose $X$ has well-defined scattered $\Pi_{1}$-products rel. $H \leq \pi_{1}\left(X, x_{0}\right)$, i.e. $\mathrm{Cl}_{\text {spp }}(H)=H$. Then $\mathrm{Cl}_{W_{A}, w_{\infty}}(H)=H$ for $A=\left\{\frac{n-1}{n} \mid n \in \mathbb{N}\right\} \cup\{1\}$. By Lemma 6.2 we have $\mathrm{Cl}_{C, c_{\infty}}(H)=\mathrm{Cl}_{W_{A}, w_{\infty}}(H)=H$, showing that $H$ is $\left(C, c_{\infty}\right)$ closed. For the converse, suppose $H$ is $\left(C, c_{\infty}\right)$-closed. Fix a closed scattered set $A \subseteq[0,1]$ containing $\{0,1\}$. We check that $H$ is $\left(W_{A}, w_{A, \infty}\right)$-closed. Suppose $f:\left(\mathbb{W}_{A}, w_{0}\right) \rightarrow\left(X, x_{0}\right)$ is a map such that $f_{\#}\left(W_{A}\right) \leq H$. We will prove that $f_{\#}\left(w_{A, \infty}\right)=f_{\#}\left(\left[v \cdot \lambda^{-}\right]\right) \in H$.

Identify $A$ with the subspace $A \times\{0\}$ of $\mathbb{W}_{A}$. Since $F\left(\mathbb{W}_{A}, A\right)=1$ if 0 is a limit point of $A$, we must consider an alternative test space. Let $\mathbb{Y}$ be the space obtained by attaching a 1-cell $L_{I}$ to $\mathbb{W}_{A}$ with boundary $\left\{w_{0}, a\right\}$ for each $I=(a, b) \in \mathcal{I}(A)$ with $a>0$. We give $\mathbb{Y}$ the weak topology with respect to the subspaces $\mathbb{W}_{A}$ and $L_{I}$ for $I=(a, b) \in \mathcal{I}(A)$ with $a>0$. Although $\mathbb{Y}$ will not always be metrizable, $\mathbb{Y}$ is homotopy equivalent to a one-dimensional subspace $\mathbb{Y}^{\prime} \subset \mathbb{R}^{3}$ by a bijective homotopy equivalence $\mathbb{Y} \rightarrow \mathbb{Y}^{\prime}$ which maps $\mathbb{W}_{A}$ to $\mathbb{W}_{A} \times\{0\}$ and each 1-cell $L_{I}$ to an arc of diameter $\geq 1$ in $\mathbb{R}^{3}$ that connects $(0,0,0)$ to $(a, 0,0)$ for each $I=(a, b) \in \mathcal{I}(A)$ with $a>0$. Therefore, we may apply results for one-dimensional metric spaces to $\mathbb{Y}$.

Let $i: \mathbb{W}_{A} \rightarrow \mathbb{Y}$ be the inclusion map and $\rho_{I}:[0,1] \rightarrow \mathbb{Y}$ be the arc from $w_{0}$ to $a=\inf (I)$ along the attached 1-cell $L_{I}$. Define $g: \mathbb{Y} \rightarrow X$ so that $\left.g\right|_{\mathbb{W}_{A}}=f$ and $\left.g \circ \rho_{I} \equiv v\right|_{[0, a]}$ for $I=(a, b) \in \mathcal{I}(A), a \neq 0$. The continuity of $g$ is clear since $\mathbb{Y}$ has the weak topology. Notice that $\left[v \cdot \lambda^{-}\right] \in \operatorname{Sc}(\mathbb{Y}, A)$.

First, observe that $g_{\#}(F(\mathbb{Y}, A)) \leq H$. Since a detailed proof of this inclusion requires a straightforward but somewhat tedious decomposition of the elements of $F(\mathbb{Y}, A)$, we give a separate proof below in Lemma 6.7. Recalling the existence of a bijective homotopy equivalence between $\mathbb{Y}$ and a one-dimensional metric space, 
we have $\operatorname{Sc}(\mathbb{Y}, A)=\mathrm{Cl}_{C, c_{\infty}}(F(\mathbb{Y}, A))$ by Theorem 5.10. Combining this with the inclusion $g_{\#}(F(\mathbb{Y}, A)) \leq H$ and the closure properties from Lemma 3.3, we have

$$
\begin{aligned}
f_{\#}\left(w_{A, \infty}\right)=g_{\#}\left(\left[v \cdot \lambda^{-}\right]\right) & \in g_{\#}(\operatorname{Sc}(\mathbb{Y}, A)) \\
& =g_{\#}\left(\mathrm{Cl}_{C, c_{\infty}}(F(\mathbb{Y}, A))\right) \\
& \leq \mathrm{Cl}_{C, c_{\infty}}\left(g_{\#}(F(\mathbb{Y}, A))\right) \\
& \leq \mathrm{Cl}_{C, c_{\infty}}(H) \\
& =H
\end{aligned}
$$

where the last equality holds since $H$ is $\left(C, c_{\infty}\right)$-closed.

Lemma 6.7. Consider the spaces, maps, and groups as constructed in the proof of Theorem 6.6. Then $g_{\#}(F(\mathbb{Y}, A)) \leq H$.

Proof. Recalling that $f_{\#}\left(W_{A}\right) \leq H$, it suffices to show $g_{\#}(F(\mathbb{Y}, A)) \leq f_{\#}\left(W_{A}\right)$. To prove this inclusion, we identify a set of generators for $F(\mathbb{Y}, A)$ using a few cases. Recall that $g \circ \rho_{I}=f \circ v_{[0, a]}$ whenever $I=(a, b) \in \mathcal{I}(A)$ with $a>0$.

Case I: In the case that 0 is an isolated point of $A$, there is a maximal $a \in(0,1] \cap A$ such that $[0, a] \cap A$ is finite. In this case, $\mathbb{W}_{A} \cap([0, a] \times[0,1 / 2])$ is a finite graph which is the union of $[0, a] \times\{0\}$ and finitely many semicircles $C_{I}, I \in \mathcal{I}([0, a] \cap A)$. Thus $\pi_{1}\left(\mathbb{W}_{A} \cap([0, a] \times[0,1 / 2]), w_{0}\right)$ is freely generated by $\left\{w_{I} \mid I \in \mathcal{I}([0, a] \cap A)\right\}$. Hence if $\alpha:[0,1] \rightarrow \mathbb{W}_{A} \cap([0, a] \times[0,1 / 2])$ is a loop based at $w_{0}$, then $g_{\#}([\alpha]) \in$ $g_{\#}\left(i_{\#}\left(W_{A}\right)\right)=f_{\#}\left(W_{A}\right)$.

Case II: Suppose $I=(a, b) \in \mathcal{I}(A)$ where $a>0$ and $b$ is not an isolated point of A. Then

$g_{\#}\left(\left[\rho_{I} \cdot v_{[a, b]} \cdot \lambda_{[a, b]}^{-} \cdot \rho_{I}^{-}\right]\right)=\left[\left(\left.f \circ v\right|_{[0, b]}\right) \cdot\left(f \circ \lambda_{[a, b]}\right)^{-} \cdot\left(\left.f \circ v\right|_{[0, a]}\right)^{-}\right]=f_{\#}\left(w_{I}\right) \in f_{\#}\left(W_{A}\right)$.

Case III: Suppose $I=(a, b) \in \mathcal{I}(A)$ where $a>0$ and $b$ is an isolated point of $A$. Then we may write $J=(b, c) \in \mathcal{I}(A)$. We have $g_{\#}\left(\left[\rho_{I} \cdot v_{[a, b]} \cdot \rho_{J}^{-}\right]\right)=1$ and $g_{\#}\left(\left[\rho_{I} \cdot \lambda_{[a, b]} \cdot \rho_{J}^{-}\right]\right)=f_{\#}\left(w_{I}^{-1}\right) \in f_{\#}\left(W_{A}\right)$.

Case IV: Fix (possibly equal) $I=(a, b)$ and $J=(c, d)$ in $\mathcal{I}(A)$ where $A \cap$ $[\min \{a, c\}, \max \{b, d\}]$ is finite. Consider any reduced loop of the form $\alpha=\rho_{I} \cdot \beta \cdot \rho_{J}^{-}$ where $\beta$ is a finite concatenation of paths of the form $v_{K}, \lambda_{K}, v_{K}^{-}$, or $\lambda_{K}^{-}$for $K \in$ $\mathcal{I}(A)$. Observe that $\alpha$ is homotopic to a finite concatenation of loops from either Case II or Case III. Hence, $g_{\#}([\alpha])$ factors as a product of elements in the subgroup $f_{\#}\left(W_{A}\right)$ and must therefore be in that subgroup.

For the general case, let $\alpha:[0,1] \rightarrow \mathbb{Y}$ be a loop based at $w_{0}$ such that $[\alpha] \in$ $F(\mathbb{Y}, A)$. Since $\mathbb{Y}$ is one-dimensional, we may assume $\alpha$ is reduced and, thus, that $\alpha^{-1}(A)$ is finite. If $a$ is a limit point of $A$, then no subpath of $\alpha$ can cross the line $x=a$ within $\mathbb{W}_{A}$. Therefore, $\alpha$ is a reparameterization of a finite concatenation $\prod_{i=1}^{m} \alpha_{i}$ of loops $\alpha_{i}:[0,1] \rightarrow \mathbb{Y}$ based at $w_{0}$ where either

(1) $\alpha_{i}$ is a reparameterization of a finite concatenation of paths of the form $v_{K}, \lambda_{K}, v_{K}^{-}$, or $\lambda_{K}^{-}$for $K \in \mathcal{I}(A)$ (this can only occur if 0 is an isolated point of $A$ ) as described in Case I.

(2) $\alpha_{i}$ is a reparameterization of $\rho_{I} \cdot \beta_{i} \cdot \rho_{J}^{-}$for intervals $I=(a, b)$ and $J=(c, d)$ in $\mathcal{I}(A)$ (where the two are possibly equal) where $A \cap[\min \{a, c\}, \max \{b, d\}]$ is finite and $\beta_{i}$ is a reparameterization of a finite concatenation of paths of the form $v_{K}, \lambda_{K}, v_{K}^{-}$, or $\lambda_{K}^{-}$for $K \in \mathcal{I}(A)$. In this situation, $\alpha_{i}$ is a loop described by Case IV. 
In any of these cases (for all $i$ ), we have $g_{\#}\left(\left[\alpha_{i}\right]\right) \in f_{\#}\left(W_{A}\right)$ and, thus, $g_{\#}([\alpha]) \in$ $f_{\#}\left(W_{A}\right)$.

Remark 6.8. Finally, we note how the proof of Theorem 1.2 is established from the above results. Let $(*)$ represent the property: "the trivial subgroup of $\pi_{1}\left(X, x_{0}\right)$ is $\left(C, c_{\infty}\right)$-closed." Theorem 5.12 gives the equivalence of $(*)$ with (1) and (2) for arbitrary spaces and with (5) if $X$ is first countable. The last two equivalences also hold for arbitrary spaces: Lemma 6.2 in the case $H=1$ is $(*) \Leftrightarrow(3)$ and Theorem 6.6 in the case $H=1$ is $(*) \Leftrightarrow(4)$.

\section{A REMARK ON DENSE PRODUCTS}

We conclude with a brief remark on products over non-scattered order types. In light of Theorem 1.2, it is natural to ask if the well-definedness of products indexed over any countable linear order, including dense orders, is also equivalent to the homotopically Hausdorff property.

Definition 7.1. Let $X$ be a path-connected space. We say that $X$ has

(1) well-defined transfinite $\pi_{1}$-products if for any $x \in X$ and maps $f, g:\left(\mathbb{H}, b_{0}\right) \rightarrow$ $(X, x)$ from the Hawaiian earring such that $f \circ \ell_{n} \simeq g \circ \ell_{n}$ for each $n \in \mathbb{N}$, then $f_{\#}=g_{\#}$.

(2) well-defined transfinite $\Pi_{1}$-products if for any paths $\alpha, \beta:[0,1] \rightarrow X$ that agree on a closed set $S \subset[0,1]$ containing $\{0,1\}$ and such that $\left.\alpha\right|_{[a, b]}$ is pathhomotopic to $\left.\beta\right|_{[a, b]}$ for each component $(a, b)$ of $[0,1] \backslash S$, then $[\alpha]=[\beta]$ in $\Pi_{1}(X)$.

The above definitions appear in 2 as Definitions 3.17 and 7.1 respectively, where they are both characterized in terms of closure operators.

Question 7.2. If $X$ is a homotopically Hausdorff Peano continuum, must $X$ have well-defined transfinite $\pi_{1}$-products and/or well-defined transfinite $\Pi_{1}$-products?

\section{REFERENCES}

[1] J. Brazas, Generalized covering space theories, Theory and Appl. of Categories 30 (2015) $1132-1162$.

[2] J. Brazas, H. Fischer, Test map characterizations of local properties of fundamental groups, To Appear in the Journal of Topology and Analysis. 2019.

[3] J. Brazas, H. Fischer, On the failure of the first Čech homotopy group to register geometrically relevant fundamental group elements, Preprint. 2019. arXiv:1902.08887

[4] N. Brodskiy, J. Dydak, B. Labuz, A. Mitra, Covering maps for locally path-connected spaces, Fund. Math. 218 (2012) 13-46.

[5] J.W. Cannon, G.R. Conner, On the fundamental groups of one-dimensional spaces, Topology Appl. 153 (2006) 2648-2672.

[6] J.W. Cannon, G.R. Conner, The combinatorial structure of the Hawaiian earring group, Topology Appl. 106 (2000) 225-271.

[7] G.R. Conner, W. Hojka, M. Meilstrup, Archipelago groups, Proc. Amer. Math. Soc. 143 (2015) 4973-4988.

[8] G.R. Conner, C. Kent, Fundamental groups of locally connected subsets of the plane, Advances in Mathematics 347 (2019) 384-407.

[9] G.R. Conner, M. Meilstrup, D. Repovš, A. Zastrow, M. Željko, On small homotopies of loops, Topology Appl. 155 (2008) 1089-1097.

[10] M.L. Curtis, M.K. Fort, Jr., The fundamental group of one-dimensional spaces, Proc. Amer. Math. Soc. 10 (1959) 140-148.

[11] K. Eda, Free $\sigma$-products and noncommutatively slender groups, J. of Algebra 148 (1992), 243-263. 
[12] K. Eda, Free $\sigma$-products and fundamental groups of subspaces of the plane, Topology Appl. 84 (1998), 283-306.

[13] K. Eda, Free Subgroups of the Fundamental Group of the Hawaiian Earring, J. Algebra. 219 (1999) 598-605.

[14] K. Eda, The fundamental groups of one-dimensional spaces and spatial homomorphisms, Topology Appl. 123 (2002) 479-505.

[15] K. Eda, Homotopy types of one-dimensional Peano continua, Fund. Math. 209 (2010) 27-42.

[16] K. Eda, K. Kawamura, The fundamental groups of one-dimensional spaces, Topology Appl. 87 (1998) 163-172.

[17] H. Fischer, D. Repovš, Z. Virk, and A. Zastrow, On semilocally simply-connected spaces, Topology Appl. 158 (2011) 397-408.

[18] H. Fischer, A. Zastrow, The fundamental groups of subsets of closed surfaces inject into their first shape groups, Algebraic and Geometric Topology 5 (2005) 1655-1676.

[19] H. Fischer, A. Zastrow, Generalized universal covering spaces and the shape group, Fund. Math. 197 (2007) 167-196.

[20] H. Fischer, A. Zastrow, Combinatorial $\mathbb{R}$-trees as generalized Caley graphs for fundamental groups of one-dimensional spaces, Geometriae Dedicata 163 (2013) 19-43.

[21] A.S. Kechris, Classical Descriptive Set Theory, Springer-Verlag, New York, 1995.

[22] C. Kent, Homomorphisms of fundamental groups of planar continua, Pacific Journal of Mathematics 295 (2018) 43-55.

[23] J.G. Rosenstein, Linear Orderings, Pure and Applied Mathematics Series, Volume 98. Academic Press, New York, 1982.

[24] Z. Virk, A. Zastrow A homotopically Hausdorff space which does not admit a generalized universal covering, Topology Appl. 160 (2013) 656-666. 